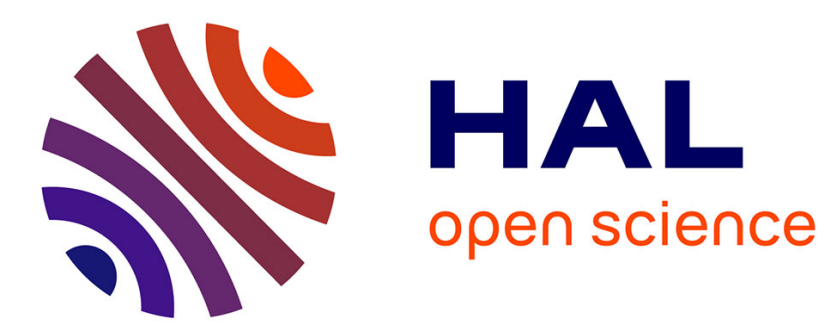

\title{
Mesure du rendement absolu de radioluminescence des scintillateurs liquides à base de benzène et de ses dérivés méthylés
}

C. Fuchs, G. Laustriat

\section{- To cite this version:}

C. Fuchs, G. Laustriat. Mesure du rendement absolu de radioluminescence des scintillateurs liquides à base de benzène et de ses dérivés méthylés. Revue de Physique Appliquée, 1970, 5 (4), pp.617-623. 10.1051/rphysap:0197000504061700 . jpa-00243434

\section{HAL Id: jpa-00243434 https://hal.science/jpa-00243434}

Submitted on 1 Jan 1970

HAL is a multi-disciplinary open access archive for the deposit and dissemination of scientific research documents, whether they are published or not. The documents may come from teaching and research institutions in France or abroad, or from public or private research centers.
L'archive ouverte pluridisciplinaire HAL, est destinée au dépôt et à la diffusion de documents scientifiques de niveau recherche, publiés ou non, émanant des établissements d'enseignement et de recherche français ou étrangers, des laboratoires publics ou privés. 


\title{
MESURE DU RENDEMENT ABSOLU DE RADIOLUMINESCENCE DES SCINTILLATEURS LIQUIDES A BASE DE BENZẼNE ET DE SES DÉRIVÉS MÉTHYLÉS
}

\author{
C. FUCHS et G. LAUSTRIAT \\ Laboratoire de Physique des Rayonnements et d'Electronique Nucléaire, \\ Centre de Recherches Nucléaires, Strasbourg - Cronenbourg, France
}

(Rę̧u le 2 février 1970)

\begin{abstract}
Résumé. - On décrit une méthode expérimentale permettant d'évaluer le rendement absolu de scintillation et on présente les résultats obtenus avec des solutions de $\alpha$-naphtyl-2 phényl-5 oxazole $(\alpha-\mathrm{NPO})$ à $5 \mathrm{~g} / 1$ dans le benzène et ses dérivés méthylés. Les valeurs trouvées $(0,020 \pm 0,002$ dans le cas du benzène privé d'oxygène) sont notablement inférieures à celles antérieurement rapportées dans la littérature. On indique également les rendements radiolytiques de formation des états excités singulets du solvant $\left(G_{\mathrm{S}_{1}}=0,98\right.$ pour le benzène) que l'on déduit de ces mesures.
\end{abstract}

\begin{abstract}
An experimental method for the determination of the absolute scintillation yield $\eta$ is presented. Values of $\eta$ obtained on deoxygenated solutions of $\alpha$-NPO in aromatic solvents $(\eta=0.020 \pm 0.002$ in the case of oxygen-free benzene) are lower than these previously reported. Radiolytic yields $G_{\mathrm{S}_{1}}$ for the formation of solvent lowest excited states $\left(G_{\mathrm{S}_{1}}=0.98\right.$ for benzene) are deduced from these results.
\end{abstract}

Introduction. - Bien que la radioluminescence des milieux organiques soit mise à profit depuis une vingtaine d'années pour la détection des rayonnements nucléaires, peu de déterminations du rendement énergétique de scintillation ont été effectuées et la plupart consistaient à évaluer celui-ci par des mesures relatives, en prenant comme référence le rendement d'un cristal ou d'une solution aromatique.

Les travaux antérieurs ont surtout porté sur le monocristal d'anthracène, pour lequel la plupart des auteurs s'accordent sur la valeur de $4 \%$ (soit 0,015 photon par électron-volt absorbé) dans le cas d'une excitation par des rayons $\beta$, ce qui correspond à un rendement radiolytique $G_{\mathrm{S}_{1}}$ de formation de l'état excité singulet $S_{1}$ responsable de la fluorescence égal à 2,2 [1-4]. D'autres cristaux organiques, tels que le stilbène, le naphtalène et le terphényle, ont également été examinés [5] et présentent des rendements inférieurs à celui de l'anthracène.

En ce qui concerne les scintillateurs liquides, Hastings et Weber [6] en 1963, ont obtenu, pour une solution dégazée de deux solutés - diphényl-2,5 oxazole-1,3 (PPO) et di(phényl-5 oxazolyl-2) benzène (POPOP) - dans le toluène, soumis aux rayons $\beta$ du carbone 14 , un rendement de $5,2 \%$, donc supérieur à celui de l'anthracène. Dans un travail plus récent, où ils comparent les intensités d'émission d'une solution de terphényle dans le benzène, excitée, soit par le rayonnement de ${ }^{14} \mathrm{C}$, soit par une radiation ultraviolette, Lipsky et coll. [7] trouvent une valeur plus faible : $4,2 \%$ - soit 0,0116 photon par eV — dans le cas idéal où le transfert d'énergie du solvant au soluté serait total et déduisent que le rendement radiolytique $G$ de formation de l'état excité ${ }^{1} B_{2 u}$ du benzène est égal à 1,55. Toutefois, la méthode utilisée par ces auteurs nécessite un certain nombre de corrections délicates (de géométrie notamment) et le carbone 14 présente l'inconvénient d'émettre un rayonnement polycinétique dans le domaine $(0-100 \mathrm{keV})$ où le rendement de scintillation varie précisément avec l'énergie de la particule $[8,9,10]$.

Comme il est important de connaître la valeur de ce rendement, tant dans l'étude du mécanisme de la radioluminescence que dans celle des effets primaires des rayonnements [8], nous nous sommes proposés d'en effectuer à nouveau la détermination, dans le cas de quelques scintillateurs liquides. La méthode expérimentale que nous avons utilisée, consiste à comparer dans les mêmes conditions, l'intensité de la radioluminescence provoquée par des électrons monocinétiques à celle de la fluorescence résultant d'une excitation du soluté par des éclairs lumineux très brefs. Après un court rappel du mécanisme de l'émission, permettant d'établir les expressions du rendement de scintillation, nous exposerons le principe de notre méthode et les conditions expérimentales de son 
application. Puis nous indiquerons la valeur du rendement absolu de scintillation obtenue pour le système benzène- $\alpha$-NPO, ainsi que celle des rendements radiolytiques de formation des états excités du benzène et de ses dérivés méthylés : toluène, p-xylène et mésitylène.

I. Expressions du rendement de scintillation. - On sait que lors du ralentissement d'une particule chargée, les molécules d'un milieu aromatique sont préférentiellement activées sur des niveaux singulets $\left(S_{n}\right)$ d'énergie élevée, puis soumises à une conversion interne non radiative vers l'état excité singulet le plus bas $\left(\mathrm{S}_{1}\right)$ [8]. Dans le cas d'un scintillateur liquide, ces deux processus, très rapides, ont lieu dans le solvant et sont suivis d'un troisième, plus lent, consistant en un transfert de l'énergie d'excitation sur les molécules de soluté ${ }^{1}$ ). Celles-ci se trouvent ainsi portées, à leur tour, à l'état $\mathrm{S}_{1}$ et émettent alors une radiation de fluorescence qui constitue la scintillation.

$\mathrm{Si}$, à la suite de ces différents processus, une particule d'énergie $E$ provoque l'excitation de $N_{F}$ molécules de soluté, de rendement quantique de fluorescence $\Phi$, le nombre $\eta_{P}$ de photons émis par unité d'énergie absorbée est donné par :

$$
\eta_{P}=N_{F} \Phi / E
$$

et l'expression du rendement énergétique $\eta_{E}$ (rapport de l'énergie de l'émission de fluorescence à celle perdue par la particule) s'écrit :

$$
\eta_{E}=\eta_{P} \times h v=N_{F} \Phi h v / E
$$

où $h$ est la constante de Planck et $v$ la fréquence moyenne de l'émission.

Par ailleurs, si $N_{\mathrm{S}_{1}}$ représente le nombre de molécules excitées de solvant produites par la particule et $\varepsilon$ l'efficacité de transfert d'énergie, on a :

$$
N_{S_{1}}=N_{F} / \varepsilon \text {. }
$$

L'énergie moyenne $W_{\mathrm{S}_{1}}$ nécessaire pour produire un état singulet excité du solvant est alors

$$
W_{S_{1}}=E / N_{S_{1}}
$$

et le rendement radiolytique $G_{\mathrm{S}_{1}}$ (nombre d'états $\mathrm{S}_{1}$ créés pour une absorption de $100 \mathrm{eV}$ )

$$
G_{S_{1}}=100 \times N_{S_{1}} / E=100 W_{S_{1}}^{-1} .
$$

Notons que le rendement de scintillation, qui s'écrit aussi

$$
\eta_{E}=G_{S_{1}} \varepsilon \Phi h v / 100
$$

dépend essentiellement du nombre d'états $S_{1}$ créés par la particule dans le solvant : en effet, les solutés

(1) Des travaux récents $[11,12]$ ont montré que pour de fortes teneurs en soluté, ce transfert peut avoir lieu à partir des états $S_{n}$ et $S_{1}$ du solvant. Dans ce travail toutefois, où les concentrations utilisées sont relativement faibles, on peut admettre que le transfert n'implique que les états excités de plus basse énergie. couramment utilisés ont des rendements quantiques $\Phi$ proches de l'unité [13], des fréquences moyennes d'émission $v$ voisines, et sont par ailleurs employés à des concentrations suffisantes pour que le transfert d'énergie soit presque total [14].

II. Principe de la méthode de mesure. - Les valeurs de $\Phi$ et de $h v$ étant connues pour la plupart des solutés - et, de plus, facilement mesurables —, la grandeur qu'il importe de déterminer, pour évaluer le rendement de scintillation $\eta_{P}$ ou $\eta_{E}$, est le nombre $N_{F}$. La méthode que nous avons adoptée pour cela comporte trois étapes.

Dans un premier temps, on soumet le scintillateur liquide à des particules d'énergie connue (électrons de conversion de ${ }^{137} \mathrm{Ba}$ ) et l'on relève à l'aide d'un sélecteur multicanaux le spectre des hauteurs d'impulsion délivrées par un photomultiplicateur (P. M.). Le pic correspondant au rayonnement monocinétique, d'énergie $E$, est dû à des impulsions électriques dont l'amplitude $A_{R}$ est donnée par :

$$
A_{R}=a \cdot N_{F} \cdot \Phi \cdot k_{R}
$$

où $a$ est un facteur de proportionnalité et $k_{R}$ l'efficacité de collection par le P. M. des photons émis dans le scintillateur.

La seconde étape consiste à soumettre le même scintillateur à des éclairs lumineux monochromatiques très brefs $\left(\approx 10^{-9} \mathrm{~s}\right)$, de longueur d'onde telle que le soluté soit excité directement dans son premier niveau d'énergie ; ainsi les éclairs de fluorescence émis ont une durée comparable à celle des scintillations. On détermine alors, dans les mêmes conditions que précédemment, l'amplitude $A_{F}$ des impulsions électriques délivrées par le P. M. En utilisant la même notation que ci-dessus, affectée ici de l'indice $F$, et en désignant par $n$ le nombre de photons absorbés dans le scintillateur par éclair d'excitation, on a :

$$
A_{F}=a \cdot n \cdot \Phi \cdot k_{F} .
$$

Le nombre $N_{F}$ de molécules de soluté excitées à la suite de l'absorption de la particule d'énergie $E$ est alors donné par

$$
N_{F^{\prime}}=n \frac{A_{R}}{A_{F}} \times \frac{k_{F}}{k_{R}} .
$$

La dernière partie de l'expérience a pour but de déterminer le rapport $k_{F} / k_{R}$ et le nombre $n$ de photons absorbés par le soluté à chaque éclair; on effectue cette dernière mesure à l'aide d'un photomultiplicateur, étalonné selon une technique que nous exposerons plus loin.

III. Conditions expérimentales. - 1. DisPositif. Les éclairs de lumière excitatrice sont fournis par un tube à décharge comportant deux électrodes en argent mouillées au mercure et placées dans une atmosphère d'hydrogène et d'argon $\left(20 \mathrm{~kg} / \mathrm{cm}^{2}\right)$ sous une tension de $15 \mathrm{kV}$. L'impulsion lumineuse, dont la durée est 
de deux à trois nanosecondes à mi-hauteur, est synchrone d'un signal électrique qui, après mise en forme, est enregistré par une échelle de comptage (Fig. 1).

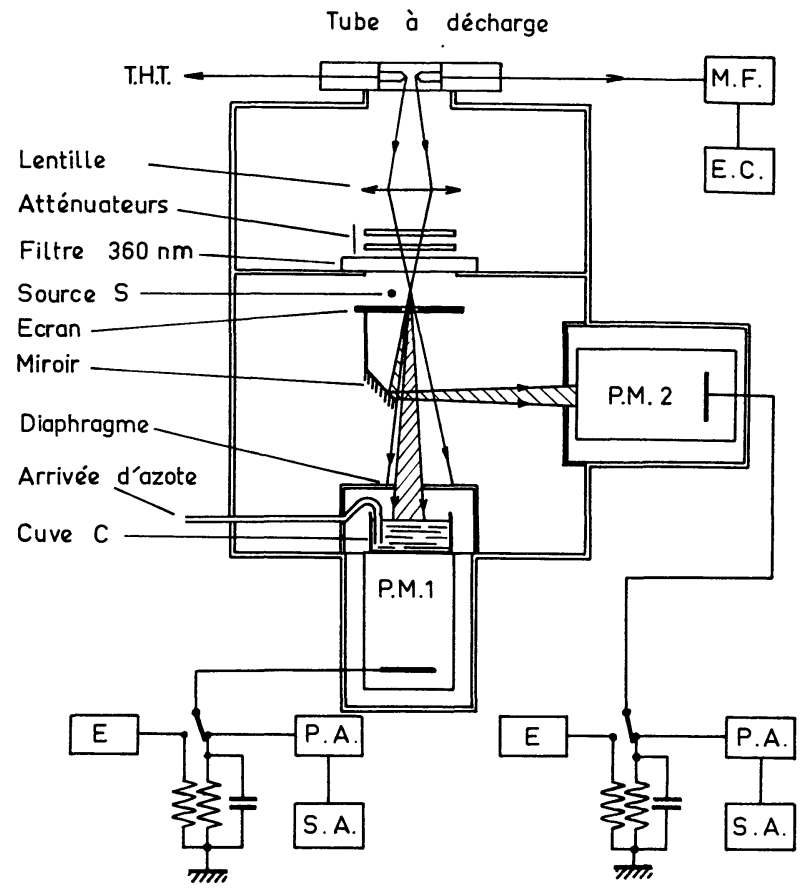

Fig. 1. - Dispositif expérimental.

E. Electromètre.

P. A. Préamplificateur.

S. A. Sélecteur d'amplitude.

M. F. Mise en forme.

E. C. Echelle de comptage.

P. M. Photomultiplicateur.

Le faisceau lumineux traverse successivement une lentille de quartz L, une série d'atténuateurs (filtres neutres de densités optiques connues) et un filtre interférentiel (centré autour de $360 \mathrm{~nm}$ ); il est ensuite focalisé en un point très voisin de l'emplacement de la source radioactive $\mathrm{S}$. La cuve $\mathrm{C}$ contenant le scintillateur, couplée optiquement à un photomultiplicateur P. M. 1. (DARIO 53 UVP), est surmontée d'un diaphragme d'ouverture fixe $\left(0,5 \mathrm{~cm}^{2}\right)$. Cette disposition assure l'égalité des angles solides sous lesquels le scintillateur est irradié par le faisceau et par le rayonnement nucléaire. Le fait que le parcours des particules dans le milieu est légèrement différent de celui des photons entraîne cependant une différence des efficacités de collection de lumière qui sera discutée plus loin.

Afin de contrôler la stabilité de l'émission du tube à décharge, une faible fraction du faisceau lumineux est réfléchie par un miroir vers un second photomultiplicateur P. M. 2 (DARIO 53 UVP). Ce miroir est solidaire d'un écran de plomb mobile qui masque la source radioactive lors de l'excitation optique et intercepte le faisceau lumineux durant l'irradiation par les particules.

Les gaz dissous dans le scintillateur, et notamment l'oxygène dont l'effet inhibiteur est bien connu, sont déplacés par passage, bulle à bulle, d'un courant d'azote.

Notons enfin que l'anode de chaque P. M. peut être connectée, soit à un électromètre à condensateur vibrant, soit à une installation de spectrométrie nucléaire (préamplificateur et sélecteur multicanaux). Dans le premier cas, on mesure un courant continu proportionnel à l'intensité du faisceau lumineux issu du tube à décharge ; la seconde connection est utilisée pour enregistrer les spectres d'amplitude des impulsions électriques.

2. Energie des Électrons. - Pour tenir compte du ralentissement des particules dans l'air et dans le film de mylar recouvrant la source de césium 137, l'énergie $E$ des électrons de conversion a été déterminée en remplaçant le scintillateur par un compteur à semiconducteur (barrière de surface or-silicium) et trouvée égale à $602 \mathrm{keV}$.

3. Composition du scintillateur. - Nous avons étudié plusieurs scintillateurs liquides, composés de différents solvants et d'un même soluté.

Les solvants employés étaient le benzène et ses dérivés méthylés : toluène, $p$-xylène et mésitylène. Bien que de qualité spectroscopique, ils ont été soumis à un passage sur colonne d'alumine et de gel de silice.

Quant au choix du soluté, il a été imposé par les conditions expérimentales. En effet, pour augmenter la précision des mesures, nous avons sélectionné, dans le spectre d'émission du tube à décharge, le domaine de plus grande intensité, situé vers 360 nm (Fig. 2). A

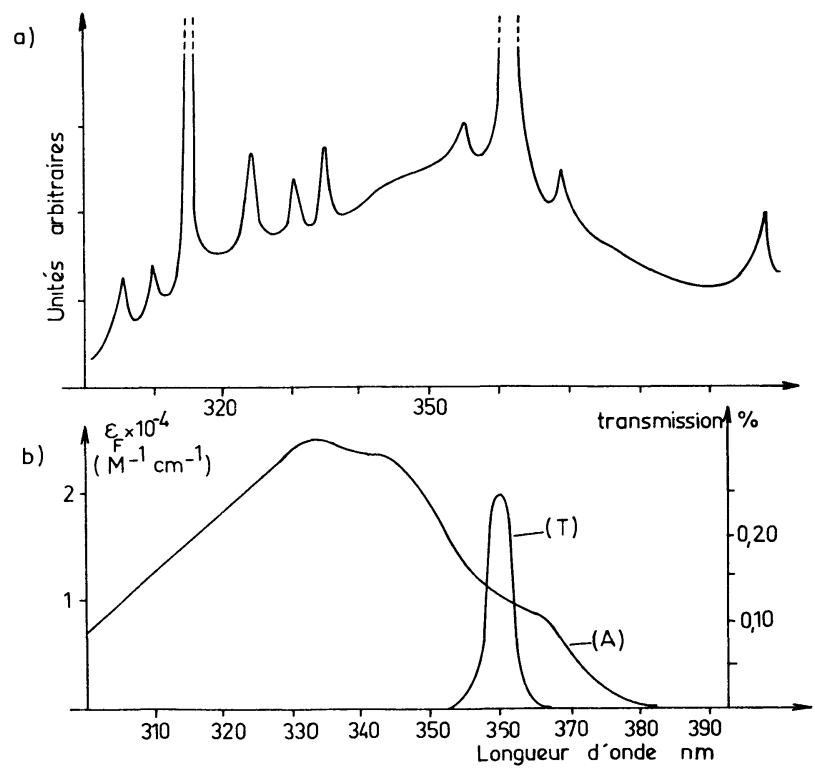

FIG. 2a. - Spectre d'émission du tube à décharge.

b. - Spectre d'absorption du soluté (A) et courbe de transmission du filtre interférentiel (T)

cette longueur d'onde, autour de laquelle est centrée la bande passante du filtre interférentiel, les quatre solvants aromatiques sont parfaitement transparents et le soluté devait présenter une bande d'absorption. 
Nous avons ainsi été amenés à retenir le 1-naphtyl-2, phényl-5, oxazole-1,3 ( $\alpha$-NPO), en l'utilisant, après purification par recristallisation dans le benzène, à une concentration suffisante $(5 \mathrm{~g} / 1)$ pour que le transfert d'énergie solvant-soluté soit important, comme nous le préciserons plus loin.

4. EtalonNage du PHOtomultiplicateur. - Le photomultiplicateur P. M. 1 a été étalonné en mesurant par actinométrie, le flux de photons produisant, dans les conditions expérimentales définies ci-dessus, un courant déterminé. Le flux issu du tube à décharge étant trop faible pour pouvoir être évalué avec précision à l'aide de la solution actinométrique, nous avons été conduits, dans cette partie de l'expérience, à remplacer ce tube par une lampe à vapeur de mercure, dont l'émission, beaucoup plus intense, est atténuée dans un rapport connu lorsqu'on mesure le courant d'anode $\mathrm{du}$ photomultiplicateur.

La détermination s'effectue en trois temps. La cuve $\mathrm{C}$ étant vide et les atténuateurs placés sur le trajet du faisceau, on note la valeur du courant d'anode. Puis on introduit dans la cuve une solution de ferrioxalate de potassium [15], on retire les atténuateurs et on irradie l'actinomètre durant un intervalle de temps donné (environ $2 \mathrm{~h}$ ). Enfin, la concentration en ions ferreux formés par photoréduction de $\mathrm{Fe}^{3+}$ est dosée par spectrophotométrie d'absorption après coloration à l'orthophénanthroline. Connaissant le rendement photochimique de la solution $(1,21$ à $360 \mathrm{~nm}$ [15]), la variation de sa densité optique, le temps d'exposition et le rapport d'atténuation, on en déduit le nombre de photons détectés par unité de temps, par le photomultiplicateur, dans la première partie de la mesure.

Le coefficient de transmission des filtres neutres (de l'ordre de 0,1 pour chacun) a été déterminé par spectrophotométrie et contrôlé à l'aide de l'installation de spectrométrie, en notant le déplacement du pic correspondant aux éclairs de fluorescence lorsqu'on introduit un atténuateur sur le trajet du faisceau.

D'autre part, nous nous sommes assurés de la validité des résultats donnés par notre solution de ferrioxalate en les comparant à ceux fournis par une autre solution actinométrique à base de leucocyanure de vert de malachite [16]. Celle-ci n'étant utilisable qu'à des longueurs d'onde inférieures à $340 \mathrm{~nm}$, nous avons irradié, lors d'une expérience annexe, les deux actinomètres à $310 \mathrm{~nm}$ et dans les mêmes conditions expérimentales : les deux séries de résultats concordaient à $2 \%$ près.

Dans nos conditions expérimentales, le courant anodique du photomultiplicateur était de $1 \mu \mathrm{A}$ lorsque $4,3 \times 10^{7}$ photons de $360 \mathrm{~nm}$ traversent le diaphragme par seconde. Ce résultat représente la valeur moyenne de douze mesures indépendantes, et est caractérisé par une " erreur probable » (c'est-à-dire telle que la probabilité d'une erreur plus grande est inférieure ou égale à 0,5 ) de $5 \%$.
IV. Détermination du rendement absolu de scintillation de la solution de $\alpha$-NPO dans le benzène. - La détermination du nombre $N_{F}$ de molécules de soluté excitées à l'état $S_{1}$ par une particule chargée nécessite la connaissance des différents paramètres de la relation (4), à savoir :

- le rapport $A_{R} / A_{F}$ des hauteurs d'impulsions dues au rayonnement nucléaire et aux éclairs lumineux,

- le rapport $k_{F} / k_{R}$ des efficacités de collection de lumière pour les deux types d'excitation,

- le nombre $n$ de photons absorbés dans le scintillateur par éclair.

1. RAPPORT $A_{R} / A_{F}$. - La figure 3 montre, à titre d'exemple, les courbes de distribution des hauteurs

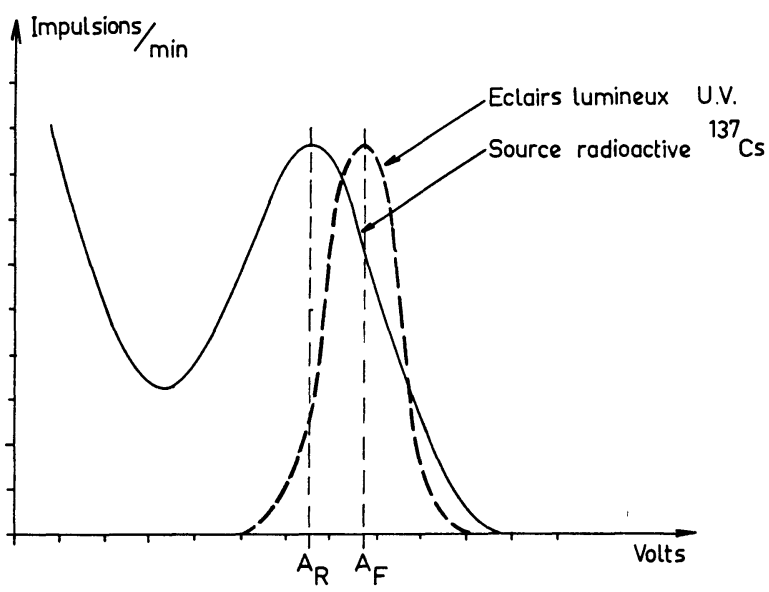

FIG. 3. - Courbes de distribution des hauteurs d'impulsion.

d'impulsions obtenues à l'aide de l'installation de spectrométrie, lorsque le scintillateur $\left(2 \mathrm{~cm}^{3}\right.$ de solution de $\alpha$-NPO ( $5 \mathrm{~g} / 1)$ dans le benzène saturé d'azote) est soumis au rayonnement de la source de ${ }^{137} \mathrm{Cs}$ et aux éclairs lumineux. Le premier spectre (courbe en trait plein) présente un pic, dû aux électrons de conversion, d'abscisse $A_{R}$. La seconde courbe (en trait discontinu), donne l'amplitude $A_{F}$ des impulsions provoquées par le tube à décharge. Dans ce cas, l'intensité des éclairs a été atténuée par les filtres neutres de façon à rendre $A_{F}$ voisin de $A_{R}$.

2. Nombre $n$. - Le nombre moyen $n$ de photons reçus par le scintillateur à chaque éclair est déterminé en retirant la solution de la cuve $\mathrm{C}$ et en mesurant simultanément le courant anodique du photomultiplicateur étalonné (P. M. 1) et le nombre de décharges délivrées par le tube par unité de temps. Le résultat obtenu doit toutefois être corrigé pour tenir compte des différences de réflexion du faisceau lumineux sur les surfaces libres de l'actinomètre et du scintillateur. Les coefficients de réflexion air-eau et air-benzène étant respectivement voisins de $2 \%$ et de $4 \%$, le facteur de correction appliqué est de 1,02.

$\mathrm{Vu}$ l'incertitude de l'étalonnage du photomultiplicateur, la valeur moyenne du produit $n \times A_{R} / A_{F}$, établie à la suite de douze expériences indépendantes, 
était de $6,25 \times 10^{3}$, avec une " erreur probable » de $8 \%$.

3. RAPPORT $k_{F} / k_{R}$. - Dans nos conditions expérimentales (Fig. 4a), le rayonnement excitateur, nucléaire ou ultraviolet, pénètre par la surface libre

a)

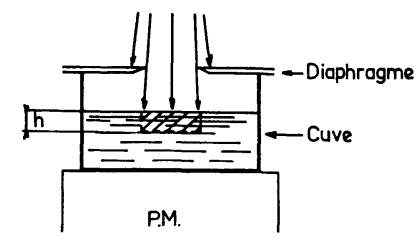

FIG. 4a. - Représentation schématique de la zone fluorescente.

de la solution et la luminescence apparaît dans un volume cylindrique dont la section est délimitée par l'ouverture du diaphragme et la hauteur $h$ égale au parcours du rayonnement dans la solution $\left({ }^{2}\right)$. Comme l'efficacité $k$ de collection de la lumière émise dans le scintillateur varie avec la distance séparant la cathode du photomultiplicateur du point d'émission [17], il était nécessaire de calculer la profondeur moyenne de pénétration $\bar{h}$ des particules et des photons, et d'examiner ensuite la variation de $k$ en fonction de $\bar{h}$.

Dans le cas de l'excitation par les électrons, le parcours de ces derniers dépend du pouvoir d'arrêt du solvant. Si l'on désigne par $I_{R}$ l'intensité d'une scintillation, le nombre de photons émis par unité de parcours $\left(\mathrm{d} I_{R} / \mathrm{d} x\right)$ est proportionnel, en chaque point de la trace, à la perte d'énergie spécifique $(\mathrm{d} E / \mathrm{d} x)$ de la particule $[8,18]$. Nous avons représenté sur la figure $4 b$ (courbe en trait plein) la variation $\mathrm{de}\left(\mathrm{d} I_{R} / \mathrm{d} h\right)$,

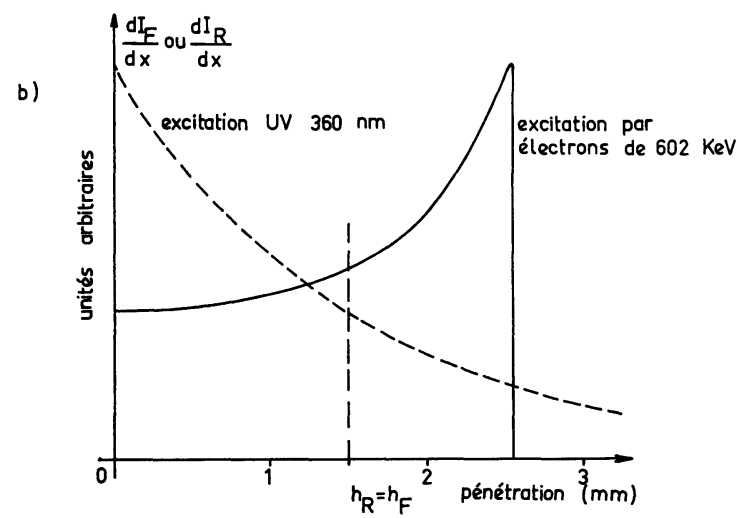

FIG. 4b. - Variation des intensités de fluorescence et de radioluminescence, en fonction de la pénétration de rayonnement incident.

c'est-à-dire celle de $(\mathrm{d} E / \mathrm{d} h)$, en fonction de la pénétration $h$ des électrons de $602 \mathrm{keV}$, dont le parcours dans

(2) Cette description ne s'applique en toute rigueur qu'au cas de l'excitation par les éclairs lumineux, dont le flux est uniformément réparti sur toute la section du volume considéré. Avec les particules, les scintillations apparaissent chacune le long de traces bien localisées, mais leurs origines sont statistiquement distribuées sur toute la surface, de sorte que l'on peut également considérer ici un volume moyen d'émission. le benzène est de $2,60 \mathrm{~mm}$ [19]. On peut évaluer à partir de cette courbe la profondeur moyenne $\bar{h}_{R}$ à laquelle apparaît la radioluminescence

$$
\bar{h}_{R}=\frac{\int_{0}^{\infty} h \cdot \frac{\mathrm{d} I_{R}(h)}{\mathrm{d} h} \mathrm{~d} h}{\int_{0}^{\infty} \frac{\mathrm{d} I_{R}(h)}{\mathrm{d} h} \mathrm{~d} h}=1,5 \mathrm{~mm} .
$$

Considérons maintenant le cas de l'excitation par les éclairs lumineux, produisant des éclairs de fluorescence d'intensité $I_{F}$. Le nombre de photons émis $\left(\mathrm{d} I_{F} / \mathrm{d} h\right)$ à une distance $h$ de la surface dépend $\mathrm{du}$ nombre de photons incidents absorbés à ce niveau ; il décroît selon une loi exponentielle

$$
\left(\frac{\mathrm{d} I_{F}}{\mathrm{~d} h}\right)=K \exp \left(-2,3 \varepsilon_{F}[F] h\right)
$$

où $K$ est une constante et $\varepsilon_{F}$ le coefficient d'extinction molaire de $\alpha-\mathrm{NPO}$ à $360 \mathrm{~nm}$. La profondeur moyenne d'émission $\bar{h}_{F}$ est ici donnée par

$$
\bar{h}_{F}=\frac{\int_{0}^{\infty} h K \mathrm{e}^{-2,3 \varepsilon_{F}[F] h} \mathrm{~d} h}{\int_{0}^{\infty} K \mathrm{e}^{-2,3 \varepsilon_{F}[F] h} \mathrm{~d} h}=\frac{1}{2,3 \varepsilon_{F}[F]} .
$$

Sachant que $\varepsilon_{F}=1,084 \times 10^{4} \mathrm{M}^{-1}$ à cm $\mathrm{cm}^{-1} 360 \mathrm{~nm}$, on en déduit que la teneur en soluté nécessaire pour que $\bar{h}_{F}=\bar{h}_{R}=1,5 \mathrm{~mm}$, est de $7,25 \times 10^{-2} \mathrm{~g} / 1$ (Fig. $4 b$, courbe en pointillés). A cette concentration, l'efficacité de collection de lumière est donc pratiquement identique pour les deux modes d'excitation :

$$
k_{F}(0,0725 \mathrm{~g} / 1) \approx k_{R} \text {. }
$$

A la teneur de $5 \mathrm{~g} / 1$, la pénétration des photons est plus faible et l'efficacité de collection $k_{F}(5 \mathrm{~g} / 1)$ est moindre. On détermine aisément la variation de $k_{F}$ en comparant, à l'aide du dispositif décrit plus haut, les intensités des éclairs de fluorescence fournis par les deux solutions, dans le cas d'une excitation optique. Nous avons trouvé :

$\frac{A_{F}(5 \mathrm{~g} / 1)}{A_{F}(0,0725 \mathrm{~g} / 1)}=\frac{k_{F}(5 \mathrm{~g} / 1)}{k_{F}(0,0725 \mathrm{~g} / 1)}=\frac{k_{F}}{k_{R}}=0,87 \pm 0,02$.

4. Rendement de SCintillation. - Ces différentes données expérimentales permettent de calculer le nombre $N_{F}$ de molécules de soluté excitées par un électron de $602 \mathrm{keV}$ dans le scintillateur étudié :

$$
N_{F}=(5,45 \pm 0,55) \times 10^{3} .
$$

Connaissant la valeur du rendement quantique de fluorescence de $\alpha-\mathrm{NPO}(\Phi=0,7[13])$ et l'énergie moyenne des photons émis par ce soluté $(h v=$ $3,06 \mathrm{eV}$ ), on déduit le rendement de scintillation de la solution étudiée, en absence d'oxygène :

$$
\eta_{E}=0,020 \pm 0,002
$$


et le nombre de photons émis par unité d'énergie absorbée :

$$
\eta_{P}=(6,4 \pm 0,7) \times 10^{-3} \text { photon } / \mathrm{eV} .
$$

Ces deux valeurs sont notablement plus faibles que celles rapportées antérieurement par les autres auteurs [1-7]. Elles ne peuvent toutefois pas leur être directement comparées puisqu'elles ont été obtenues avec un scintillateur dont le soluté ( $\alpha$-NPO) est différent, les caractéristiques de ce composé intervenant comme nous l'avons indiqué plus haut - par la valeur du rendement quantique de fluorescence et par celle de la fréquence moyenne d'émission.

V. Détermination du rendement radiolytique de formation des états $S_{1}$ dans le benzène et ses dérivés méthylés. - Des résultats précédents, on déduit immédiatement le nombre $N_{\mathrm{S}_{1}}=N_{F} / \varepsilon$ de molécules excitées de benzène susceptibles de transmettre leur énergie au soluté, si l'on connaît la valeur du coefficient de transfert $\varepsilon$.

Nous avons évalué ce paramètre, dans le cas d'une excitation par des particules, en mesurant les hauteurs d'impulsion $A_{R}$ pour des solutions de concentration $[F]$ variable et en examinant la variation de $1 / A_{R}$ en fonction de $1 /[F]$. On sait, en effet, que la hauteur d'impulsion est proportionnelle au coefficient de transfert, lequel dépend de la teneur $[F]$ selon la relation

$$
\frac{1}{\varepsilon}=1+\frac{1}{\alpha[F]}
$$

où $\alpha$ est constant pour un scintillateur donné [14]. En normalisant à l'unité l'ordonnée à l'origine de la droite expérimentale, on obtient la courbe donnant la valeur de $1 / \varepsilon$ en fonction de $1 /[F]$. Pour une concentration de $5 \mathrm{~g} / 1$ en $\alpha$-NPO dans le benzène saturé d'azote, on trouve en particulier $\varepsilon=0,92 \pm 0,02$ et le nombre $N_{\mathrm{S}}$ recherché est alors :

$$
N_{\mathrm{S}_{1}}=(5,9 \pm 0,6) \times 10^{3} .
$$

On en déduit l'énergie moyenne $W_{\mathrm{S}_{1}}$, requise pour créer un état singulet $S_{1}$ d'une molécule de benzène :

$$
W_{\mathrm{S}_{1}}=E / N_{\mathrm{S}_{1}}=(102 \pm 10) \mathrm{eV}
$$

et le rendement radiolytique de formation d'un tel état

$$
G_{\mathrm{S}_{1}}=0,98 \pm 0,1 \text {. }
$$

Nous avons étendu notre travail à des solutions de $\alpha$-NPO, à même concentration $(5 \mathrm{~g} / 1)$, dans les solvants homologues du benzène : toluène, $\mathrm{p}$-xylène et mésitylène. Le soluté étant identique dans tous les cas, ainsi que le facteur de correction $k_{F} / k_{R}$, les rendements de scintillation de ces solutions sont obtenus à partir de celui du benzène par simple comparaison des hauteurs d'impulsion. Les résultats sont indiqués dans les deux premières colonnes du tableau $\mathrm{I}$; on note que les solvants les plus efficaces sont le p-xylène et le mésitylène, ce qui avait déjà été signalé par plusieurs auteurs
[20]. Ayant également mesuré, pour ces systèmes, le coefficient $\varepsilon$ de transfert d'énergie, nous avons évalué l'énergie de formation $W_{\mathrm{S}_{1}}$ des états $\mathrm{S}_{1}$ des divers solvants étudiés, ainsi que leurs rendements radiolytiques $G_{\mathrm{S}_{1}}$. Les valeurs trouvées sont reportées dans les dernières colonnes du tableau $\mathrm{I}$.

\section{TABLEAU I}

$\begin{array}{cccccc}\begin{array}{c}\text { Solvant } \\ \text { saturé } \\ \text { d'azote }\end{array} & \begin{array}{c}\eta_{E} \\ (\%)\end{array} & \begin{array}{c}\eta_{P} \\ (h v / \mathrm{eV})\end{array} & \varepsilon & \begin{array}{c}W_{\mathrm{S}_{1}} \\ (\mathrm{eV})\end{array} & G_{\mathrm{S}_{1}} \\ - & - & - & - & - & - \\ \text { Benzène } & 2,0 & 6,35 \times 10^{-3} & 0,92 & 102 & 0,98 \\ \text { Toluène } & 2,4 & 7,65 \times 10^{-3} & 0,93 & 85 & 1,18 \\ \text { p-Xylène } & 2,55 & 8,10 \times 10^{-3} & 0,92 & 79 & 1,27 \\ \text { Mésitylène } & 2,55 & 8,10 \times 10^{-3} & 0,93 & 81 & 1,24\end{array}$

Caractéristiques de scintillateurs renfermant $5 \mathrm{~g} / 1$ de $\alpha$-NPO dans différents solvants aromatiques privés d'oxygène. (L'incertitude sur ces valeurs est de l'ordre de $10 \%$ ).

VI. Discussion des résultats. - En analysant les données expérimentales de Hastings et Weber [6], Lipsky et coll. ont montré que le rendement de formation de l'état $S_{1}$ qui s'en déduisait, pour le toluène excité par le rayonnement $\beta$ du carbone 14, était de l'ordre de 1,9 , ce qui correspond à une énergie de formation $W_{\mathrm{S}_{1}}$ d'environ $53 \mathrm{eV}$ [8]. Ces auteurs trouvent eux-mêmes pour le benzène dégazé et irradié par ${ }^{14} \mathrm{C}, W_{\mathrm{S}_{1}}=64,5 \mathrm{eV}$ soit $G_{\mathrm{S}_{1}}=1,55$. Ces deux valeurs de $G_{\mathrm{S}_{1}}$ sont très supérieures à celles que nous obtenons pour les mêmes solvants. Une légère différence entre les résultats précités et les nôtres aurait pu être attribuée à l'énergie des particules : on sait, en effet, que le rendement de scintillation - et donc la valeur de $G_{\mathbf{S}_{1}}$ - diminue pour des électrons de faible énergie $(E \leqslant 100 \mathrm{keV})[8,9,10]$. Mais l'importance de l'écart (de l'ordre de $50 \%$ ) entre les deux séries de résultats ne nous semble explicable que par des raisons d'ordre expérimental.

Deux considérations plaident en faveur des valeurs trouvées dans ce travail :

- Les rendements absolus de scintillation obtenus par Hastings et Weber et par Lipsky et coll. sont respectivement de $5,2 \%$ et $4,2 \%$. Ils se trouvent ainsi l'un et l'autre supérieurs à la valeur couramment admise par le monocristal d'anthracène $(4,0 \%)$ reconnu comme le meilleur scintillateur organique, et peuvent donc être suspectés d'erreurs par excès.

- Dans une étude antérieure, Pringle et coll. [21] ont signalé que l'efficacité de scintillation d'une solution de p-terphényle et de diphényl-2,5 oxazole-1,3 (PPO) dans le p-xylène, était de $30 \%$ inférieure à celle du cristal d'anthracène, ce qui correspondrait à $0,0105 h v / e V$. Or, dans une telle solution, le coefficient de transfert d'énergie est proche de l'unité, et le rendement quantique de fluorescence de PPO est de 0,75 [13] : on peut donc en déduire que l'énergie de 
formation $W_{\mathbf{S}_{1}}$ doit être de l'ordre de $72 \mathrm{eV}$ pour le p-xylène, valeur en accord avec celle $(79 \pm 10 \mathrm{eV})$ que nous obtenons.

Quant aux variations de $W_{\mathrm{S}_{1}}$ et de $G_{\mathrm{S}_{1}}$ avec la nature $\mathrm{du}$ solvant, elles s'interprètent bien si l'on admet, comme nous l'avons fait ici, qu'à la concentration relativement faible en soluté utilisée, la majeure partie du transfert d'énergie a lieu à partir du niveau excité le plus bas du solvant. En effet, le nombre $N_{\mathrm{S}_{1}}$ d'états $\mathrm{S}_{1}$ créés par la particule dépend du nombre $N_{\mathrm{S}_{n}}$ d'états primaires $S_{n}$ de haute énergie, initialement engendrés par celle-ci, et de l'efficacité $\beta$ de conversion interne $\mathrm{S}_{n} \rightarrow \mathrm{S}_{1}\left(N_{\mathrm{S}_{1}}=N_{\mathrm{S}_{n}} \times \beta\right)$. Or, s'il est admis que la section efficace de formation des niveaux $S_{n}$ est sensiblement identique pour tous les solvants organiques $[22,23]$, des travaux récents $[24,25]$ ont montré que l'efficacité de conversion interne $\beta$ croît avec le nombre de groupements méthyles fixés sur le noyau aromatique. On doit donc s'attendre à ce que le rendement $G_{\mathrm{S}_{1}}$ augmente du benzène au p-xylène, ce que nous observons effectivement.
Conclusion. - La méthode mise au point pour mesurer le rendement de scintillation des solutions organiques nous semble plus précise que celles employées dans les travaux antérieurs, car la radioluminescence y est provoquée par un rayonnement monocinétique et la comparaison des intensités des scintillations et des éclairs de fluorescence qu'elle implique, ne nécessite qu'une faible correction d'effet géométrique, qui est d'ailleurs facile à évaluer. Les valeurs des rendements absolus de scintillation obtenues par cette méthode $(2,0 \%$ pour une solution de $\alpha$-NPO dans le benzène saturé d'azote), sont inférieures à celles rapportées dans la littérature. Les rendements radiolytiques $G_{\mathrm{S}_{1}}$ des solvants aromatiques étudiés, ont été trouvés voisins de 1,0 (benzène) - 1,2 (toluène et mésitylène) et 1,3 (para-xylène). Cette technique est actuellement étendue à d'autres scintillateurs ainsi qu'à l'étude des monocristaux organiques.

Nous tenons à remercier Monsisur le Professeur A. Coche, Directeur du Laboratoire, pour ses conseils et pour l'intérêt qu'il a porté à ce travail.

\section{Bibliographie}

[1] Birks (J. B.), « The Theory and Practice of Scintillation Counting )) Pergamon Press, Londres, 1964.

[2] Birks (J. B.), Szendrei (M. E.), Phys. Rev., 1953, 91, 199.

[3] Sangster (R. C.), Irvine (J. W.), J. Chem. Phys., 1956, 24, 670 .

[4] Butt (P. K.), Proc. Phys. Soc., 1953, A 66, 940.

[5] Chizhikova (Z. A.), Opt. Spectry. (URSR) English Tran., 1959, 7, 176.

[6] Hastings (J. W.), Weber (G.), J. Opt. Soc. Amer., $1963,53,1410$

[7] Skarstad (P.), Ma (R.), Lipsky (S.), Mol. Crystals, $1968,4,3$.

[8] Voltz (R.), " Actions Chimiques et Biologiques des Radiations )) (M. Haissinsky, éditeur), Masson, Paris, 1969.

[9] Brannen (E.), Olde (G. N.), Rad. Res. Rev., 1962, $16,1$.

[10] HopkINS (J. I.), Rev. Sci. Instr., 1951, 22, 29.

[11] Voltz (R.), Rad. Res. Rev., 1968, 1, 301.

[12] Klein (J.), Thèse Sc. Phys., Strasbourg, 1968.

[13] Lami (H.), Laustriat (G.), J. Chem. Phys., 1968, 48, 1832.
[14] Tanielian (C.), Thèse Sc. Phys., Strasbourg, 1965.

[15] Hatchard (G. G.), Parker (C. A.), Proc. Roy. Soc., 1956, A 235, 518.

[16] Calvet (J. C.), Rechen (J. L.), J. Amer. Chem. Soc., 1952, 74, 2101.

[17] Coche (A.), Laustriat (G.), J. Phys. Rad., 1958, 19, 595.

[18] Da Silva (J. L.), Thèse Sc. Phys., Strasbourg, 1969.

[19] Nuclear Sciences Series no 23, Nat. Ac. of Science, Washington, 1964.

[20] Laustriat (G.), Voltz (R.), Klein (J.), Symposium on the current status of liquid scintillation counting, M. I. T. Cambridge, 1969.

[21] Pringle (P. W.), Black (L. D.), Funt (B. L.), Sobering (S.), Phys. Rev., 1953, 92, 1582.

[22] Platzman (R. L.), Rad. Res., 1962, 17, 419.

[23] Skebele (A.), Lassettre (E. N.), J. Chem. Phys., 1965, 42, 395.

[24] Braun (C. L.), Kato (S.), Lipsky (S.), J. Chem. Phys., 1963, 39, 1645.

[25] Lawson (C. W.), Hirayama (F.), Lipsky (S.), $J$. Chem. Phys., 1969, 51, 1590. 